Voix et Images

voixetimages

\title{
La recherche du pays chez Paul-Marie Lapointe et Gérald Godin - Concerto pour arbres
}

\section{Cécile Pelosse}

Volume 1, numéro 1, septembre 1975

Hubert Aquin

URI : https://id.erudit.org/iderudit/013987ar

DOI : https://doi.org/10.7202/013987ar

Aller au sommaire du numéro

Éditeur(s)

Les Presses de l'Université du Québec

ISSN

0318-9201 (imprimé)

1705-933X (numérique)

Découvrir la revue

Citer cet article

Pelosse, C. (1975). La recherche du pays chez Paul-Marie Lapointe et Gérald

Godin - Concerto pour arbres. Voix et Images, 1(1), 80-88.

https://doi.org/10.7202/013987ar d'utilisation que vous pouvez consulter en ligne.

https://apropos.erudit.org/fr/usagers/politique-dutilisation/ 


\section{La recherche du pays chez Paul-Marie Lapointe et Gérald Godin - Concerto pour arbres}

En 1960, Paul-Marie Lapointe publie “Arbres ${ }^{1}$ », un grand poème qui fait date et où il exorcise la peur ancestrale des forêts primitives qui pesait sur l'imaginaire québécois. Il appelle chaque arbre par son nom et la forêt anonyme, terrifiante, se laisse pénétrer, devient accueillante et familière.

Ce long poème qui s'étale sur huit pages suggère une forêt de mots arbres, organisés en grandes familles, avec des blancs qui figurent l'espace entre chaque arbre et d'autres qui ménagent des clairières. L'ensemble se présente comme une suite de variations sur le thème central. Il se déploie en trois grands mouvements dont chacun est consacré à une catégorie d'arbres. Dans un premier temps, défilent les conifères, puis viennent les feuillus, bouleau en tête, peuplier à l'arrière-garde, enfin toute espèce d'arbres nourriciers, s'offrant avec leurs écorces, leurs feuilles ou leurs fruits, pour la faim et la soif des oiseaux, des bêtes et des hommes. Le choix que le poète fait des essences américaines, son penchant pour les réalités sensibles, l'ordonnance de l'ensemble où les caprices de la fantaisie font bon ménage avec les exigences de la logique, maintes trouvailles, enfin, font de ce poème une œuvre personnelle.

Point de syntaxe élaborée, mais une organisation de type primitif, fondée sur la parataxe et la juxtaposition de phrases nominales. Ce sont rarement les verbes qui lient les mots entre eux, mais plutôt les affinités, évidentes ou secrètes, qui unissent les choses et les êtres, identifiés aux mots. Cette volonté de confondre le nom et l'arbre ressort des premiers mots: “j'écris arbre", qui rappellent la manière quasi magique de Césaire dans Cadastre ( (Je dis fleuve ${ }^{2}$ ), visant à capter la chose dans le mot. L'absence d'article transforme un nom commun en prénom. Comme on dit Pierre, au lieu d'une pierre ou de la pierre, le poète appelle chaque arbre par son nom, tantôt nom de famille, «pins blancs», "cèdre de l'est", "épinettes grises", "sapins blancs", tantôt prénom singulier lorsque l'évocation s'y prête, à cause de son sens unique, précis : 
L'originalité la plus apparente du poème, sorte de composition musicale, relève de sa structure rythmique apparentée à la musique de jazz. Est-ce un hasard si les "épinettes grises" lui font penser à quelques-uns des instruments de jazz?

\section{épinette breuvage d'été piano droit tambour fougueux}

C'est l'hiver et le temps des conifères. On pense à l'été futur en buvant de la bière d'épinette. À l'abri dans les maisons, on joue ou on écoute de la musique. Une suggestion de l'épinette, petit clavecin remplacé par le piano. L'épinette que sa bonne résonance fait rechercher pour la fabrication des tables d'harmonie. Une nouvelle correspondance entre bois et piano droit appelle le tambour: tinya ou bombo des Incas, fait de troncs d'arbres évidés, tambour des Noirs... Quant au clairon, il se fait désirer pour venir à son heure, avec les fortes images qui accompagnent le final du premier mouvement, culminant sur un point d'orgue:

conifères d'abondance espèces hérissées crêtes
vertes des matinaux scaphandriers du vent co-
nifères dons quichottes sans monture sinon la
montagne clairons droits foudroyant le ciel
conifères flammes pétrifiées vertes brûlantes
gelées de feu coniferes
arêtes de poissons verticaux dévorés par l'oiseau

Les vers libres s'organisent en versets et en strophes de dimension variable, les courts alternant avec les longs. Leurs attaques respectives, vigoureusement marquées par une invocation, compensent l'impression de désordre née d'une typographie non traditionnelle. Les rappels d'un même nom d'arbre, en cours de strophe, délimitent des unités rythmiques de dimension variable. Ainsi le rythme de la strophe sur l'aune est fondé sur une alternance de brèves et de longues, comme la poésie des Anciens: une brève: «aunes vertes», une longue: «aunes à bourrelets... raies échouées", quatre fois deux brèves: "aune fragile aux clous"..., une longue: "aune crispé "...

On pourrait aussi transcrire cette structure en blanches, noires, croches et doubles croches. Mais ce jeu de solfège ne saurait rendre compte des résonances propres à chaque mot, ni de la saveur des vocables, ni de la vérité des associations. Le son et le sens, indissociables comme en toute vraie poésie, représentent les deux principaux réseaux de correspondance. Les calembours foisonnent: «aubier " appelle «aube», «peuplier baumier " suggère «embaumer», «noyers" «noyade heureuse», "orme", "arme". Parfois, seul le sens relie un mot à un autre, comme dans l'expression «pommier croqueur». Il arrive encore qu'ensemble son et sens justifient une association qui en paraît d'autant plus heureuse: «tilleul tisane de minuit». Une répétition peut aussi se combiner avec une allitération: "érable à sucre érable source". Vérité de surcroît, puisque la sève d'où l'on tire le sirop doré et le brun sucre d'érable, lorsqu'elle sort de l'arbre, coule d'abord transparente comme l'eau d'une source. 
Si l'on reconnaît les goûts d'un homme, chacun peut goûter les richesses qu'il étale, suffisamment liées à l'expérience commune:

bouleau merisier jaune et ondé bouleau flexi-
ble acajou sucré bouleau merisier odo-
rant rouge bouleau rameau de couleuvre
feuille-engrenage vidé bouleau cambrioleur à
feuilles de peuplier passe les bras dans les cages
du temps captant l'oiseau captant le vent

Tout foisonnant qu'il soit, ce poème parlerait même à un enfant, car c'est en toute simplicité qu'il donne à regarder, à écouter, à toucher, à respirer et à goûter. A s'étonner aussi, mais comme on joue à la devinette pour le plaisir de chercher, sinon de trouver:

sapins grandissimes sapins de Babel

coiffeurs des saisons pilotis des villes fantasques

locomotives gercées toit des mines

A vrai dire, la clé des énigmes se trouve cachée par l'Imprimeur de la reine entre les pages d'un livre de géographie forestière paru en 1961: Arbres indigènes du Canada ${ }^{3}$. Le poème part de là, même si le poète y butine à sa fantaisie, en s'inspirant non seulement du texte (noms d'arbres, caractéristiques, usages) mais aussi des photos qui l'illustrent. “Genévrier qui tient le plomb des alphabets». Bois de genévrier recherché pour entourer la mine de plomb des crayons:

Peuplier faux-tremble trembleur à grands crocs

peuplier-loup griffon troubleur

D'où sort cet animal fabuleux sinon de son semblable, le dragon, voisin de dictionnaire de drageon? Et quand on sait que "le peuplier à grandes dents (...) se reproduit par drageons", on est moins troublé par ce «griffon troubleur", étonnant tout de même.

L'apparition de “têtes franciscaines" parmi les chênes n'a rien de surprenant, si l'on songe que, dans un esprit proche de celui de François d'Assise et de son Cantique des créatures, le poète chante ici son frère l'arbre. Ces "têtes franciscaines" apportent une note d'humour, à cause d'un gland «inséré à moitié de sa longueur dans une cupule profonde (...) couverte de grandes écailles formant frange à la partie supérieure ${ }^{4}$ ». Évocation malicieuse d'un crâne tonsuré, auréolé d'un bourrelet de cheveux à la franciscaine, encore que le picot au sommet de la calvitie, rappelle qu'il s'agit bien d'un gland de chêne. La reproduction d'un gracieux assemblage de fruits et de feuilles du châtaignier d'Amérique inspire ce juste commentaire poétique: "châtaignier marronnier fruiteur aux envols de drapés à stries". A l'image d'un rameau d'hiver, correspond «pattes d'oiselle rachitique», aux samares de l'érable, "parachuteurs d'ailes".

La feuille de l'orme blanc devient "scie grugeuse de vent". De l'union des feuilles de peupliers avec ses chatons pendants, sort «langues de chattes". Le saule pleureur, dont "la graine minuscule et légère porte une aigrette", évoque un coq: "graine-coq à aigrette". Lé charme 
de Caroline, qui se plaît au bord des cours d'eau, rappelle "narcisse" et le sassafras, un «roi-mage», par le truchement de l'huile de sassafras et des odeurs aromatiques de l'écorce, des rameaux et des feuilles de cet arbre. Les trochets de fruits du noyer noir semblent sortir des mains de quelques "fabriquants de boules" et le bouleau à papier qui se reconnaît à "ses branches retombantes " prend des airs d' “albatros ».

Si le poète se laisse souvent guider par l'ordonnance du livre, il opère un choix significatif à partir de l'essentiel. Entre l'ancien nom et le nom actuel de telle ou telle essence, il opte pour le nom le plus commun. Qu'il soit l'ancien nom "érable barré» - au tronc rayé — préféré à érable de Pennsylvanie, "orme liège " préféré à orme de Thomas, cormier préféré à sorbier d'Amérique. Ou qu'il soit le nom actuel, «pin à feuilles tordues», plus français et plus imagé que "pin de Murray». II n'oublie pas «bois d'orignal», appellation populaire de l'érable barré, dont les feuilles servent de "nourriture d'été " aux chevreuils et aux orignaux.

L'organisation interne des trois grands mouvements est complexe, à cause d'un entrecroisement de thèmes mineurs qui s'annoncent, se combinent progressivement au thème majeur, avant de s'effacer devant d'autres, quitte à reparaître plus tard. L'amour et la mort, le mal, la lumière et l'eau, les saisons, les bêtes sauvages et les animaux domestiques, la vie quotidienne et les activités humaines pacifiques (métiers) ou guerrières, tous ces thèmes secondaires se greffent sur le thème principal. Ils s'y confondent même si bien qu'il devient impossible de penser à l'arbre en termes abstraits. Patiente initiation au pays, totalité vivante, ce beau poème étonnamment concret, déborde largement le thème de l'arbre, épine dorsale du poème.

Le principe d'unité, c'est un jeu subtil de correspondances. Tous les sens sont à la fête mais aussi l'esprit et le cœur. On passe naturellement des formes aux couleurs, aux instruments de musique, aux senteurs, aux boissons et aux mets. Les salés, les acides et les sucrés, avec une préférence pour ces derniers. Ces sensations composent cinq chaînes d'association imbriquées les unes dans les autres et liées au thème majeur.

On lit ce poème comme on revient du Nord au Sud sans quitter le sol canadien. Et sa lecture fait vivre environ une année. II commence en hiver avec la neige et s'achève en automne, à la saison des fruits, laissant le lecteur au sein d'une heureuse surabondance. L'introduction, brève, salue en passant la sève et les racines, comme les fondations invisibles de la forêt. Puis la parole s'installe dans la lumière du plein jour, qui met en valeur les formes et les couleurs:

arbres d'orbe en cône et de sève en lumière racines de la pluie et du beau temps terre animée

Quant aux thèmes secondaires, la façon dont ils se greffent sur le thème de l'arbre, relève d'un art très sûr. En moins de deux pages, espace qu'il faut au premier mouvement, tous les thèmes mineurs sont ébauchés, présentés sous un angle suggestif, intégrés dans le groupe des conifères. À l'hiver correspond l'évocation des intérieurs paisibles: 
pins des calmes armoires et des maisons pauvres bois de table et de lit bois d'avirons de dormants et de poutres portant le pain des hommes dans tes paumes carrées

Poussé par le désir d'assumer tous les aspects de la vie, le poète associe aux "balais" et aux "aiguilles couturières", image de la vie quotidienne, les «mâts fiers voiles tendues sans remords et sans larmes " qui évoquent les grands départs. L'hiver symbolise l'austérité des débuts, temps d'âpres luttes, mais "lambris des chaleurs" rappelle qu'alors on savait vivre. "Coffres des fiançailles» amorce le thème de l'amour, tout en proposant une comparaison discrète entre ce passé et le temps des fiançailles. Fiançailles d'un peuple avec une terre, évoquées sur le ton ému d'un vieux couple fidèle qui se souvient.

Quant au mal, il est planté au centre du poème sous la forme de la croix qui sépare ici les arbres sans truits comestibles des arbres nourriciers. Parce que l'aune de l'Oregon se travaille facilement et prend bien les clous, voilà déclenchée une série d'associations spontanées:

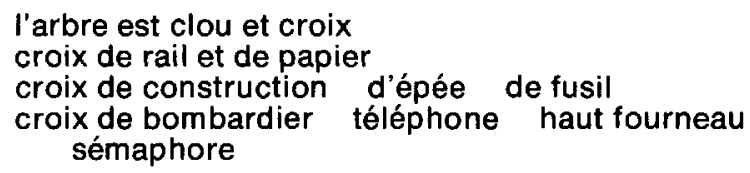

On passe d'une allusion à la mort du Christ à l'évocation des guerres, pour aboutir à une méditation sur la double postulation de l'homme moderne. Capable d'élever des gratte-ciel comme de mener une vie de "chien", de dresser les croix de néon et de pratiquer la torture, directement comme les sauvages, ou indirectement sur ses frères humains qu'il laisse mourir de faim:

croix d'aluminium et de néon

croix de gratte-ciel et de chien de torture et de faim

Et pourtant l'abondance est là, célébrée au cours du troisième mouvement, aussi long que les deux premiers réunis. Copieux menu offert en l'heureuse saison des récoltes, depuis celle du sirop d'érable au printemps jusqu'aux «labours d'automne», promesse des suivantes. Le poème s'achève sur l'espérance de voir les arbres de vie nourrir tous les enfants de la terre:

les arbres sont couronnés d'enfants tiennent chauds leurs nids sont chargés de farine dans leur ombre la faim sommeille et le sourire multiplie ses feuilles

Accord final de toute beauté. Dans cette large fresque, rien de pressant, aucune éloquence insistante, mais une troupe légère de mots égrenés comme des notes jouées au piano. La délicatesse du propos, la grâce des évocations, le charme des esquisses apparentent cette poésie transparente au meilleur Saint-Denys Garneau, dans ses aquarelles d'arbres par exemple. Poésie pure dans la veine d'Éluard, que Paul-Marie Lapointe 
rejoint dans son goût d'une poésie impersonnelle et naturellement portée aux chants d'amour. Poésie souriante, nourrie de l'expérience commune, attentive aux plus humbles réalités, servante du merveilleux qui dort dans l'ordinaire.

Voilà un bel exemple d'incarnation du verbe français où le sens pratique populaire et le pragmatisme anglo-saxon s'allient à l'esprit de contemplation sereine, à la joie de connaître et de nommer le monde.

\section{«RETABLE» DE GÉRALD GODIN}

Aux nobles “Arbres" du montréalais d'adoption Paul-Marie Lapointe, à cette œuvre séminale qui fait date, Gérald Godin, natif de TroisRivières, réplique narquoisement par son long «Retable ${ }^{5}$ ", le plus élaboré de ses Nouveaux Poèmes. Pour ce faire, il a déniché chez le même Imprimeur de la reine, un autre ouvrage, moins attrayant que la source littéraire d' «Arbres", mais aussi savant: les Mauvaises herbes du Canada ${ }^{6}$. Et voilà notre provincial qui fait son herbier en puisant à pleines brassées parmi les végétaux des sous-bois qui sont, pour ainsi dire, les prolétaires des grands bois.

“Retable» s'apparente à «Arbres" par la richesse verbale, la précision, la saveur des mots et leur assemblage harmonieux. Mais les différences sautent aux yeux. Godin insiste moins sur les fruits. II signale vaguement “les fruits amers", s'intéresse plus à leurs formes qu'à leur saveur:

ni le noyer cendré ni ses trochets de fruits

II ne manque pas une occasion de montrer l'envers du décor, le danger que présente telle plante:

l'herbe à perles

la voyante la massue la vénéneuse la narcotique

ni le seneçon de Saint-Jacques le toxique

Les désignations peu flatteuses mais pittoresques lui plaisent:

ni l'herbe aux teigneux ni la rhubarbe au diable

Les sols signalés: «remblais», "buttes de sable», «fondrières", sont plutôt pauvres et ingrats:

ni la mousse ni la fausse giroflée

du soleil si peu familières

ni la crusgalle ni la florifère

la luisante l'arrondie la foncée l'aplatie

des terrains vagues des fossés

ni la folle avoine la lâche la retombante

et son retard à germer

La manière de Godin, élégante ou solennelle, guindée ou familière, fait office d'une sorte de quant-à-soi. Le poète se protège de l'attendrissement par l'anonymat des formules et le recul critique. 
On pourrait pousser plus avant le parallèle en s'attardant aux correspondances de détail. Des fragments de "Retable" font écho à des passages d' "Arbres", à cause de leur rencontre inévitable sur le terrain des botanistes et de leur source livresque commune: Arbres indigenes du Canada. L'écart que chaque poète fait à partir de l'appellation courante est significatif de l'esprit qui l'anime. A propos de l'hamamélis, PaulMarie Lapointe met en valeur le bon usage de cette plante médicinale:

hamamélis coupant le sang des blessures

Usage que Godin passe sous silence, tout au plaisir de télescoper deux noms curieux, dont les sons se répondent:

\section{ni l'hamamélis ni l'amélanchier}

«Retable» se présente comme un très long poème de vingt et une strophes où s'étale une végétation touffue d'arbres, d'arbustes, de plantes et de fleurs, énumérés ou croqués sur le vif et peuplés de menu gibier: lièvres, sarcelle, col-vert, poulette grasse, bécassine, pluvier et quelques insectes: stellaire, grillon, criquet. En fait d'oiseaux, une chauve-souris.

Les vers libres sont disposés en strophes d'inégale longueur. Le poète use de rimes ou d'assonances avec une certaine fantaisie. Le rythme repose sur plusieurs modes de répétitions. Fréquentes reprises de " ni " en tête de vers, énumération des espèces, usage systématique de l'article défini singulier, accumulation de déterminatifs. Jeu d'assonances et d'allitérations à l'intérieur des vers: «l'enivré hivernant». Groupements alternativement binaires ou ternaires:

ni le saule à feuille de pêcher

le blanc le fragile le pleureur

ni le sarrasin de Tartarie et sa dormance

l'Ouest exposé les convois

de contreforts

les arbustes rampants les branches rabattues

Les verbes, rares, n'apparaissent que dans quelques petites relatives et à la fin. La texture du poème est faite de substantifs flanqués de déterminatifs: adjectifs, participes, génitifs ou compléments marquant la manière. L'impression de foisonnement vient de l'enchevêtrement de tous ces végétaux et de leurs qualifications: titres de noblesse:

ni le sapin concolore des sols limoneux

le royal le décidé le couronné le résineux

ou appellations roturières:

la bardane la moldavique

l'herbe de pauvreté la terne l'humiliée

A cette profusion s'ajoutent la mention de quelques signes du zodiaque, de références aux saisons, de brefs aperçus du terrain.

Le titre qui rappelle ces ornements de bois contre lesquels est adossé un autel, invite à considérer ce poème comme la transposition littéraire d'un retable richement sculpté. Le style est moins classique que baroque, à cause d'une tendance au décoratif, de la prolifération des 
essences et de l'insistance des déterminatifs. Motifs de broderie destinés à enjoliver un nom commun:

\author{
le sureau bleu \\ le glauque le balsamé \\ le raide le rugueux le caduque
}

Le goût du bizarre et de l'étrange, relève également d'une esthétique baroque:

ni le sumac à vernis le millepertuis ni l'ellébore noir

Godin procède à la manière d'un décorateur attentif aux qualités plastiques des végétaux:

ni le charbon de Russie

le recourbé le rigide l'épineux

ni l'orge agréable la finette

ses soies longues et ses soies courtes

Le son compte plus que le sens. L'écriture s'écarte moins de la source littéraire que dans “Arbres». On saisit parfois mal le lien entre les éléments respectifs de chaque strophe, et la division en strophes assez monotones ne paraît guère justifiée. Un découpage différent changerait-il beaucoup l'effet général? L'usage systématique de l'article défini singulier, évoquant chaque espèce, la prolifération des épithètes de nature et le processus d'énumération apparentent ce poème à un catalogue, avec les risques d'ennui que suppose le genre. Toutefois cette monotonie, tempérée de fantaisie, devient significative à la fin :

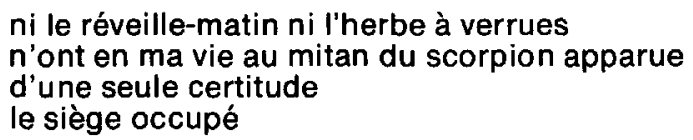

Les attaques négatives et pressantes à coups de “ni " répétés laissent pressentir un malaise. Mais chemin faisant, lancé dans son inventaire, le poète se pique au jeu avec un plaisir évident, se permettant quelques pointes de préciosité (inversions). Le poème prend à la fin des airs de grand herbier figé sur le papier. Tel un collectionneur désappointé de n'avoir fait qu'épingler les éléments d'un monde vivant, le poète voit l'essentiel lui échapper sans pouvoir le nommer. L'essentiel, c'est-à-dire la vie. L'incertitude intérieure rend vain le spectacle le plus raffiné. "Arbres" maîtrisait l'abondance pour l'usage et la joie de tous. Ici le poète s'avoue dépossédé devant une abondance qui distrait agréablement son regard, mais ne l'aide guère à vivre mieux ${ }^{7}$.

Cécile Pelosse

1. Le Réel absolu (Poèmes 1948-1965), Montréal, Éditions de l'Hexagone, 1971.

2. Aimé Césaire, Cadastre, Poèmes, Paris, Seuil, 1961, p. 83, et dans Cahier d'un retour au pays natal, p. 40: "Je dirais arbre".

3. Canada, Ministère des Forêts, Arbres indigènes du Canada, Imprimeur de la reine, 1961. 
88 voix et images

4. Ibid., p. 161.

5. Gérald Godin, Nouveaux Poèmes, Trois-Rivières, Bien public, 1964, p. 46-53.

6. Clarence Frankton, les Mauvaises herbes du Canada, Ministère de l'Agriculture du Canada, Ottawa, 1963.

7. N.D.L.R.: Cet article est extrait de la thèse inédite de madame Pélosse. intitulée la Recherche du pays dans la poésie québécoise 1945-1970. L'ouvrage a été présenté et déposé à l'Université de Montréal en juin 1974. 